\title{
Delay in Treatment of a Bimalleolar Ankle Fracture during Coronavirus Disease-19 COVID-19 Pandemic Leading to Amputation
}

\author{
Peter Regala ${ }^{1}$, Peter B White ${ }^{1}$, Adam D Bitterman ${ }^{1}$, Gus Katsigiorgis ${ }^{2}$, Paul A Dicpinigaitis ${ }^{3}$
}

Learning Point of the Article:

Public perception surrounding COVID-19 has created a sense of fear among some patients which may impact behaviors, such as prevent them from seeking prompt medical care when necessary, and the medical community must work to make patients feel safe and increased availability to patient care to prevent adverse outcomes.

\section{Abstract}

Introduction: Severe acute respiratory syndrome coronavirus-2 (SARs-CoV-2), also known as coronavirus disease-19 (COVID-19), is a novel respiratory disease which has fundamentally disrupted societal norms. Common responses to the pandemic have called for "social distancing" and national lockdowns. There is little evidence as to how public perception surrounding exposure to the virus may impact patient care. Here, we present a case of a patient who deferred medical treatment due to concerns regarding exposure to the virus.

Case Report: A 99-year-old female who presented to an emergency department of a community hospital on Long Island, New York, during the COVID-19 pandemic complaining of right ankle pain after a fall three 3 weeks ago. Fear of contracting COVID-19, led to initial avoidance of medical evaluation. On presentation, the patient presented with a Gustillo-Anderson Grade III A, bimalleolar ankle fracture with associated gangrene. After evaluation and a thorough discussion with the patient and her family, the patient underwent guillotine amputation.

Conclusion: Public perception surrounding COVID-19 has created a sense of fear that can impact patient behaviors, such as prevent them from seeking medical care when necessary. As physicians, we need to make patients feel safe and increase availability to patient care during this time to prevent similar issues as demonstrated in this case.

Keywords: Coronavirus disease-19, bimalleolar ankle fracture, amputation.COVID-19, Bimalleolar Ankle Fracture, Amputation

\section{Introduction}

Severe acute respiratory syndrome (SARS) coronavirus-2, also known as coronavirus disease-19 (COVID-19), is a novel respiratory disease which was first reported in December 2019 in Wuhan, China [1]. Since that time, COVID-19 has spread across the globe and was officially declared a pandemic on March 11, 2020, by the World Health Organization [2]. Due to the large size and scope of the pandemic, we have seen fundamental societal disruptions that have changed the framework of health care.

In response to the pandemic, we have seen extensive measures taken by countries and institutions in hopes of mitigating transmission and ensuring public safety from the virus.
Although exact protocols vary, common pieces of advice are to self-isolate for 7-14 days if one displays symptoms of COVID19 and to "socially distance" from those who are not displaying symptoms [3]. In the United States, we have seen several states make executive orders to this effect. In New York state, largely considered to be the epicenter for the virus in the United States [3], executive orders such as "New York State on PAUSE [4]," called for "social distancing" and temporarily closed all nonessential businesses in an attempt to decrease the transmission of COVID-19. Although effective in mitigating transmission, little is known as to how such efforts may impact public perception, social responses, and ability to access care during the pandemic.

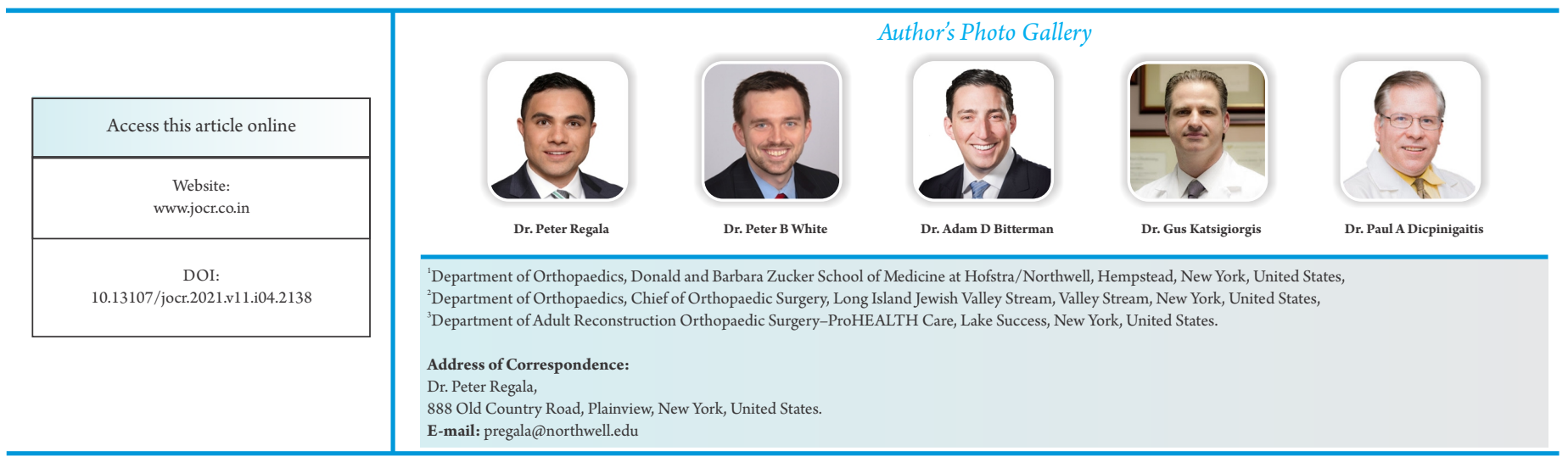

Journal of Orthopaedic Case Reports | pISSN 2250-0685 | eISSN 2321-3817 | Available on www.jocr.co.in | doi:10.13107/jocr.2021.v11.i04.2138 This is an Open Access article distributed under the terms of the Creative Commons Attribution Non-Commercial License (http://creativecommons.org/licenses/by-nc/3.0) which permits unrestricted non-commercial use, distribution, and reproduction in any medium, provided the original work is properly cited. 


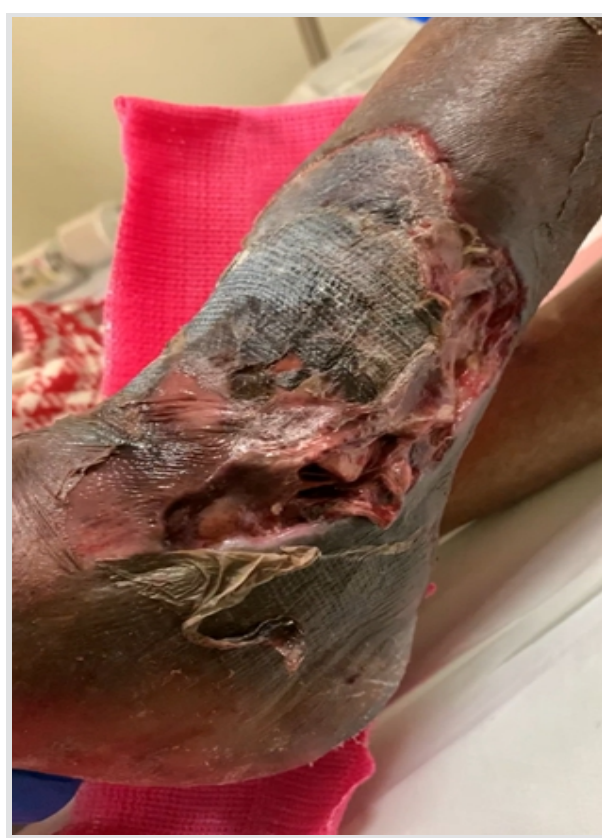

Figure 1: Clinical photograph of the right ankle demonstrate large open wound with exposed tibia.

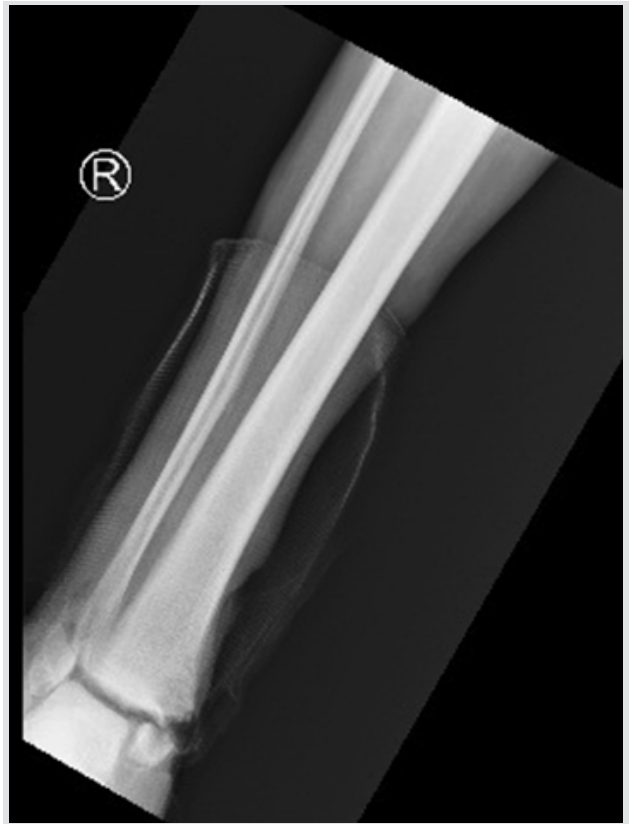

Figure 2: Initial anteroposterior radiograph of the ankle in the emergency room.
One unique feature of viral pandemics is that they can instill fear across large populations [5]. As fear is associated with clinical phobias and social anxiety disorders [6], viral pandemics can lead to widespread fear and mental distress that can manifest in various ways. One such proposed manifestation is that patients may defer or delay seeking medical evaluation or treatment during the pandemic due to fear of being exposed to COVID19. This can be evidenced as several studies have started to notice a decrease in emergency room visits for non-COVID patients visits $[7,8]$. Furthermore, a preliminary study out of China reported that patients with acute myocardial infarctions presented with larger ST segment elevations during COVID-

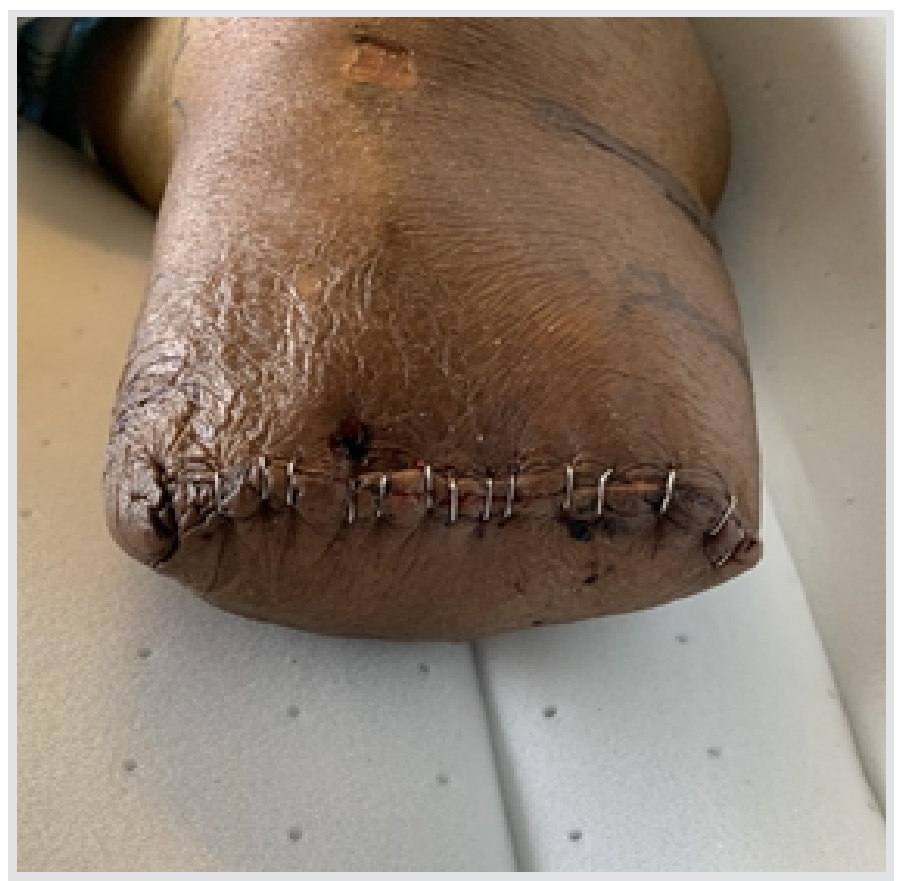

Figure 4: Clinical photo after a below-knee amputation.
19, which they attributed to delay in presentation [9]. To the best of our knowledge, there are no reports of delayed presentation secondary to COVID-19 in the orthopedic literature.

This case report describes a patient who deferred medical evaluation and treatment during the COVID-19 pandemic over concerns of exposure to the virus and ended up with a suboptimal outcome. In this case, we discuss the detrimental impact the delay in management had on the patient's outcome as well as possible mitigation strategies to reduce patient fear.

\section{Case Report}

A 99-year-old African-American female with a medical history of dementia, diabetes mellitus, hypertension, and a cerebral vascular accident with right-sided weakness who presented to an emergency department (ED) in Long Island, New York, on April 24, 2020, with the chief complaint of the right ankle pain. The patient was accompanied by her granddaughter who reported that the patient had a series of the falls over the past several weeks. She first fell 3 weeks before presentation at which time she complained of the right ankle pain. Due to concerns regarding COVID-19 exposure, especially in light of the patient's son passing away from complications of the disease, the patient did not seek further medical evaluation. The granddaughter who was also a nurse placed the ankle in a home fashioned posterior slab splint and she proceeded to weightbear as tolerated.

Two days before presentation, the patient had another fall but again did not seek medical evaluation. On the day of presentation, the granddaughter was concerned regarding the 
patient's skin overlying the ankle due to a malodorous wound prompting the evaluation. On examination, the patient had a temperature of 36.7 , blood pressure of $111 / 65$, heart rate of 82 beats/min (bpm), respiration rate of $18 \mathrm{bpm}$, and oxygen saturation of $94 \%$ on room air. There was a malodorous $20 \mathrm{~cm}$ area of necrosis on the anterior aspect of the distal tibia with a 10 $\mathrm{cm} \times 5 \mathrm{~cm}$ open wound overlying the lateral malleolus (Fig. 1). The wound was explored and distal fibula, tibia, talus, and peroneal tendons were noted. The ankle was tender to palpation about both the medial and lateral ankle with significant bony instability. Sensation and motor strength of the right lower extremity including ankle and foot were unable to be assessed due to the patient's current mental status. The foot was edematous with overlying blistering. Dorsalis pedis and posterior tibial pulses were not appreciated by palpation, with a +1 dorsalis pedis pulse on Doppler.

Laboratory testing demonstrated an initial white blood cell count of $12.67 \mathrm{~K} / \mu \mathrm{L}(3.8-10.5 \mathrm{~K} / \mu \mathrm{L})$, erythrocyte sedimentation rate of $97 \mathrm{~mm} / \mathrm{h}(0-22 \mathrm{~mm} / \mathrm{h})$, and C-reactive protein of $36.82 \mathrm{mg} / \mathrm{dL}(0.00-0.40)$. Blood cultures had growth in both aerobic and anaerobic bottles with Gramnegative rods (Morganella morganii). COVID-19 polymerase chain reaction testing was negative.

Anteroposterior, lateral, and oblique radiographs taken in the ED showed a bimalleolar ankle fracture with a transverse fracture line about the medial malleolus and an oblique fracture line about the lateral malleolus (Fig. 2, 3). Air and gas were noted in the anterior margin of the tibiotalar joint. The patient's clinical examination was consistent with an open right ankle bimalleolar fracture with presumed osteomyelitis of the right lower extremity and a gangrenous foot. On secondary examination, the patient had no other injuries.

A lengthy discussion was had with the health-care proxy about the patient's diagnosis, prognosis, and treatment options, especially limb salvage versus amputation. The health-care proxy initially wanted to attempt lower extremity salvage, but after discussion regarding overall success of salvage and associated morbidity versus amputation, the health-care proxy agreed to amputation. The following day, the patient underwent a successful right guillotine amputation by vascular surgery, with plan for a return to the operating room for a formal belowknee amputation (BKA) once her condition stabilized. OR cultures demonstrated a polymicrobial infection with Proteus mirabilis, Klebsiella oxytoca, Enterococcus faecalis, and Stenotrophomonas maltophilia. The patient received IV antibiotics for the treatment of sepsis related to her previously infected Grade III A open right ankle bimalleolar fracture and gangrenous foot. The patient's overall condition improved with no growth on repeat blood cultures and a clean non-bleeding guillotine amputation site. The patient eventually returned to the operating room and underwent a successful formal BKA (Fig.4).

\section{Discussion}

Over the past several months, the rapid emergence of COVID19 has rapidly changed the framework of health care. Although there is evidence that public health initiatives, such as "social distancing," have been effective at mitigating the spread of the virus [10], many experts suggest that the societal disruption of the pandemic may last up to 2 years [11]. As demonstrated by this case report, the burden of COVID-19 lies beyond that of managing infection rates.

There is a growing concern among health-care professionals that patients' fear of contracting COVID-19 may result in their avoidance of seeking formal medical care for evaluation and treatment, and for those that prolong evaluation may present to EDs in more severe health $[12,13]$. In this case, elderly patient sustained a bimalleolar right ankle fracture that should have been promptly evaluated by a medical professional. The apprehension of increased COVID-19 exposure in an ED led to a delay in seeking medical care. Had this patient sought medical attention at the time of the original injury or soon thereafter the overall outcome for the patient may have been different. However, the delay in medical evaluation ultimately resulted in an open right ankle fracture, with a resultant infection, which then required lower extremity amputation as definitive treatment. As evidenced by this patient, a delay in evaluation and treatment can potentially put patients at risk for complications and worse outcomes. This is similar to the evidence from China that is reporting greater ST segment elevations during myocardial infarction in patients with delayed presentations to emergency rooms during the COVID-19 pandemic [9].

The psychological fear of COVID-19 and the impact it may have on patients seeking medical treatment may not be elucidated for quite some time; however, the SARS outbreak in 2003 can offer us some parallels of patient's anxiety regarding infection control measures and their reluctance to seek medical care $[14,15]$. There can be considerable fear among persons in the general public or specific communities where there is greater burden of infectious diseases or illnesses that may lead to fearful persons to delay seeking care.

Experiences during the SARS epidemic in 2003 have served as an example of fear within communities that were greatly affected by infectious diseases. The persons within these communities feel the high anxieties which come with public measures and restrictive practices; such as quarantine and social distancing that attempt to mitigate the spread of the contagion [15]. The effects of public service announcements, media 
coverage, and mandated closures of non-essential businesses undoubtedly work to raise awareness of the infectious disease but also heighten concern over contracting the disease. The response to people during pandemics may be to avoid associated areas with the infectious disease, such as hospitals and medical offices. The detriment to avoiding medical evaluation of a non-COVID-19-related illness may ultimately be borne to the patient leading to worse outcomes.

The COVID-19 pandemic may have a societal impact for many more months to come. Patient's will require access to care for non-COVID-19-related illnesses and injuries, but there will need to be strategies which allow for safe access to care while mitigating fears of contracting the infectious disease. Although the American Medical Association suggests that telemedicine should be an adjunct to in-patient visits [16], telemedicine has emerged during the pandemic as a point of access to care for patients who may be otherwise too fearful to seek care and a useful triaging tool for providers. For patients who visit doctor's offices, "social distancing" measures should be implemented to further decrease exposure. This can be accomplished by creative strategies such as utilizing parking lots as waiting rooms, providing patients, and providers with gloves and masks or spreading out patients more effectively. COVID-19 testing and antibody testing may also provide a turning point in changing public perception of COVID-19 and provide a clearer picture of the disease's prevalence. A more thorough understanding of the prevalence of COVID-19 in communities will allow governments and health-care systems to formulate public health recommendations [17]. Public service announcements to people to visit the ED or visit their doctor when ill or injured will help to guide patients who need care.

As the number of cases of COVID-19 begin to fall and states begin to lift restrictions in the United States, medical professionals may soon be facing a new pandemic of patients with non-COVID-19-related medical illnesses and injuries complicated by their previously deferred care. The overall impact COVID-19 may have on patient outcomes may not be determined for months or years to come. Health-care providers and the communities they serve should work to increase patient outreach and invoke measures to decrease patient fears about seeking care. These efforts to decreased fears about contracting COVID-19 will help to prevent future negative health outcomes.

\section{Conclusion}

A unique feature of viral pandemics is that they can instill fear across large populations. This fear may cause patients to delay time to medical evaluation and treatment, or they may present in a more severe state of health. Preliminary evidence from China suggests that patients are presenting with worse myocardial infarctions during the COVID-19 pandemic. We present an orthopedic case report in which fears of contracting COVID-19 lead to a delay in seeking care and an adverse outcome.

\section{Clinical Message}

Public perception surrounding COVID-19 has created a sense of fear that can impact patient behaviors, such as prevent them from seeking medical care when necessary. As physicians, we need to make patients feel safe and increase availability to patient care during this time to prevent similar adverse outcomes as demonstrated in this case.

\section{References}

1. Wang D, Hu B, Hu C, Zhu F, Liu X, Zhang J, et al. Clinical characteristics of 138 hospitalized patients with 2019 novel Coronavirus-infected pneumonia in Wuhan, China.JAMA 2020;323:1061.

2. World Health Organization Director-General's Opening Remarks at the Media Briefing on COVID-19 - 11 March 2020. World Health Organization. Available from: https://www.who.int/dg/ speeches/detail/whodirector-general-s-opening-remarks-at-the-mediabriefing-on-covid-19-11-march-2020. [Last accessed on 2020 May 02].

3. World Health Organization. Coronavirus disease (COVID19) Advice for the Public. World Health Organization; 2020. Available from: https://www.who.int/ emergencies/diseases/ novel-coronavirus-2019/advice- for-public. [Last accessed on 2020 Mar 20].

4. New York State on PAUSE. Department of Health. Available from: https://coronavirus.health.ny.gov/new-york-statepause. [Last accessed on 2020 May 20].

5. Ahorsu DK, Lin CY, Imani V, Saffari M, Griffiths MD, Pakpour AH. The fear of COVID-19 scale: Development and initial validations. Int J Ment Health Addict 2020 2020;Mar 27:1-9.

6. Krueger RF, Kotov R, Watson D, Forbes MK, Eaton NR, Ruggero $\mathrm{CJ}$, et al. Progress in achieving quantitative classification of psychopathology. World Psychiatry 2018;17:282-93.

7. Feuer W. Doctors Worry the Coronavirus is Keeping Patients Away From US Hospitals as ER Visits Drop: "Heart Attacks 
Don't Stop." CNBC. Available from : https://www.cnbc.com/2020/ 04/14/doctors-worrythe-coronavirus-is-keeping- patients-away-from-ushospitals-as-er-visits-drop- heart-attacks-dont-stop.html. [Last accessed on 2020 May 02].

8. Sullivan K. As Coronavirus Surges, Non-COVID Medical Emergencies Take a Back Seat, Putting Patients at Risk. NBCNews.com. Available from: https://www. nbcnews.com/ health/health-news/coronavirus-surgesnon-covid-medical-emergencies-take-back-seat-puttingn1175871. [Last accessed on 2020 May 02].

9. Tam CC, Cheung KS, Lam S, Wong A, Yung A, Sze M, et al. Impact of Coronavirus disease 2019 (COVID-19) outbreak on ST-segment-elevation myocardial infarction care in Hong Kong, China. Circ Cardiovasc Qual Outcomes 2020;13:e006631.

10. Courtemanche C, Garuccio J, Le A, Pinkston J, Yelowitz A. Strong social distancing measures in the United States reduced the COVID-19 growth rate. Health Aff 2020;39:1237-46.

11. Gandhi KR, Murthy KV, Prasada Rao SS, Casella F. Nonpharmaceutical interventions (NPIs) to reduce COVID19 mortality. SSRN Electron J. 2020;2020:3560688.

12. Kaeding D. During COVID-19 Crisis, Some People Opt To Delay Other Medical Care. Wisconsin Public Radio. Available from: https://www.wpr.org/during-covid-19crisis-some-people-opt-delay-other-medical-care. [Last accessed on 2020 May 08].

13. Sheth K. Perspective|Hospital Admissions for Strokes Appear to have Plummeted, a Doctor Says, a Possible Sign People are Afraid to Seek Critical Help. The Washington Post. Available from: https:// www.washingtonpost.com/ national/health-science/ hospital-admissions-for-strokesappear-to-have-plummeted-a-doctors-says-a-possiblesign-people-are-afraid-to-seek-critical-help/2020 /04/08/2048b886-79ac-1 1 ea-b6ff-597f170df8f8 story.html. [Last accessed on 2020 May 08].

14. Pappas G, Kiriaze I, Giannakis P, Falagas M. Psychosocial consequences of infectious diseases. Clin Microbiol Infect 2009; 15:743-7.

15. Person B, Sy F, Holton K, Govert B, Liang A, National Center for Inectious Diseases/SARS Community Outreach Team. Fear and stigma: The epidemic within the SARS outbreak. Emerg Infect Dis 2004;10:358-63.

16. Chaet D, Clearfield R, Sabin JE, Skimming K, Council on Ethical and Judicial Affairs American Medical Association. Ethical practice in telehealth and telemedicine. J Gen Intern Med 2017;32:1136-40.

17. Abbasi J. The promise and peril of antibody testing for COVID-19.JAMA 2020;323:1881.
Conflict of Interest: Nil Source of Support: Nil

Consent: The authors confirm that informed consent was obtained from the patient for publication of this case report
How to Cite this Article

Regala P, White PB, Bitterman AD, Katsigiorgis G, Dicpinigaitis PA. Delay in Treatment of a Bimalleolar Ankle Fracture during Coronavirus Disease19 Pandemic Leading to Amputation. Journal of Orthopaedic Case Reports 2021 April;11(4):28-32. 